\title{
SATISFACCIÓN DEL CUIDADO DE ENFERMERÍA EN UCI*
}

\section{VALIDEZ DE APARIENCIA DE TRES RESULTADOS DEL NOC}

Juan Camilo Aponte**, Ana Julia Carnillo Algarra***, Claudia Lizarazo Gómez****, Olga Lucia Silva Torres*****

\section{Resumen}

Introducción: la satisfacción con el cuidado de enfermería es un proceso abstracto cuya medición es difícil en pacientes en condiciones críticas de salud. Por ello es relevante contar con instrumentos que brinden la mayor certeza posible. Objetivo: realizar la validez de apariencia de los catorce resultados del NOC para medir la satisfacción del paciente con el cuidado de enfermería en UCI. Métodos: se realizó la validación de apariencia con la presentación de los catorce resultados a un grupo de siete personas: dos médicos intensivistas, dos enfermeras especialistas en cuidado crítico con experiencia en UCI, dos pacientes y un familiar de paciente en la UCI. Resultados: tomando como punto de corte cinco respuestas positivas de siete posibles, los resultados que tienen mayor aplicabilidad para medir la satisfacción del paciente con el cuidado de enfermería en UCI son: cuidados (3001), comunicación (3002) y seguridad (3010).

Palabras clave: satisfacción del paciente, cuidados intensivos, cuidados básicos de enfermería, estudios de validación.

Abreviaturas: NOC, nursing outcomes classification/clasificación de resultados de enfermería.

\section{ASSESSING ICU* INPATIENT SATISFACTION WITH NURSING CARE APPEARANCE OF VALIDITY OF THREE NOC RESULTS}

\begin{abstract}
Introduction: assessing satisfaction with nursing care is an abstract process which is difficult to measure in criticallyill patients. Thus, counting on instruments ensuring the best possible accuracy is relevant. Objective: conducting the appearance of validity of the 14 results of the NOC classification in order to measure ICU inpatient/users satisfaction with nursing care. Methods: the appearance of validity was performed by presenting these 14 results to a group of 7 people: $\mathbf{2}$ intensive care physicians, 2 critical care nurses with ICU expertise, 2 patients and an ICU patient family member. Results: taking five positive answers out of seven answers possible as the cut-point, the most applicable results for measuring UCI inpatient satisfaction with nursing care are: care (3001), communication (3002) and reassurance (3010).
\end{abstract}

Key words: inpatient satisfaction, intensive care, basic nursing care, validation studies.

Fecha recibido:septiembre 29 de 2011 - Fecha aceptado:diciembre 15 de 2011

* Unidad de cuidados intensivos. Concordancia de validez de apariencia de los 14 resultados del NOC para medir satisfacción del paciente hospitalizado en $\mathrm{UCl}$ con el cuidado de enfermería. Resultados de la investigación a partir de la primera publicación. '

** Magister en Epidemiología Clínica de la Pontificia Universidad Javeriana. Docente Asociado, Universidad El Bosque, Bogotá DC. Colombia. *okik

Enfermera, Universidad Nacional, magister en Administración en Salud de la Pontificia Universidad Javeriana. Profesora Titular. Fundación Universitaria de Ciencias de la Salud. Bogotá DC. Colombia.

*k*k* Enfermera especialista en Cuidado Intensivo y Gerencia para la Salud, Facultad de Enfermería,Fundación Universitaria de Ciencias de la Salud, Hospital de San José, Bogotá DC. Colombia.

****** Enfermera especialista en Cuidado Intensivo. Facultad de Enfermería, Fundación Universitaria de Ciencias de la Salud, Bogotá DC. Colombia. 


\section{Introducción}

El objeto del presente trabajo fue identificar cuáles de los catorce resultados de satisfacción descritos por el $\mathrm{NOC}^{2}$ son aplicables para medir la calidad del cuidado de enfermería en pacientes que se encuentran en UCI. Se conformó un grupo de expertos integrado por dos médicos de cuidado intensivo, dos enfermeras especialistas en cuidado del paciente crítico con conocimiento de estas unidades, dos pacientes y un familiar con la experiencia de haber estado en UCI, a quienes se les solicitó que calificaran los catorce resultados con base en los criterios definidos por Moriyama. ${ }^{3}$

En la generación de nuevo conocimiento la calidad de la medición tiene un papel muy importante, como lo plantea Ricardo Sánchez ${ }^{4}$ al afirmar que los aciertos o errores en el proceso pueden afectar la validez de los datos, quiere decir que se puede perder la correspondencia entre el resultado de la medición y la realidad del fenómeno que se está midiendo. ${ }^{5-7}$

En enfermería el interés por establecer los resultados del cuidado empezó cuando Florence Nightingale registró y analizó las condiciones sanitarias y los resultados de los pacientes en la guerra de Crimea. ${ }^{2,8,9}$ Sin embargo, los esfuerzos por evaluar la práctica de enfermería fueron esporádicos y el siguiente intento de valorar calidad en salud se encuentra en el área médica, en el reporte de 1900 cuando Ernest Codman propuso medir la calidad de la atención médica, pero solo en los años sesenta Donabedian formula un modelo para evaluar la atención en salud en el cual se destaca la estructura, el proceso y los resultados. Estos parámetros fueron utilizados en los años ochenta para realizar el Medical Outcomes Study (MOS) ${ }^{2}$ cuyas medidas se definieron y relacionaron en las categorías de: criterios de valoración clínicos, valores de laboratorio y muerte, y estado funcional dentro del cual se incluye la satisfacción con los cuidados. Este estudio es importante para enfermería porque fue uno de los primeros en tener en cuenta esta última como una dimensión del cuidado.

A mediados de la misma década se retoma la evaluación específica en enfermería con el trabajo de Aydelotte, quien utilizó los cambios de las caracterís- ticas físicas y de conducta de los pacientes para evaluar la efectividad del cuidado., ${ }^{2,10,11}$ Es en la década de los setenta cuando Hober y Zimmer ${ }^{2}$ identificaron cinco medidas de resultados: la información al paciente sobre la enfermedad y sus tratamientos, el conocimiento de las medicaciones, las habilidades de autocuidado, las conductas adaptativas y el estado de salud del paciente. En la década de los ochenta Lang y Clinton ${ }^{2}$ identificaron como categorías de resultados: el estado de salud físico, de salud mental, el funcionamiento social y físico, las actitudes del conocimiento y las actitudes sanitarias, el uso de los recursos profesionales de la salud y las percepciones del paciente sobre la calidad de los cuidados enfermeros. En forma simultánea Marek ${ }^{2}$ identifica como categorías para evaluar los cuidados enfermeros las medidas fisiológicas, psicosociales, funcionales, conductas del cliente, conocimiento del cliente, control de los síntomas, mantenimiento del hogar, bienestar, consecución de objetivos, satisfacción del paciente, seguridad, frecuencia de servicio, costo, rehospitalización y resolución de los diagnósticos enfermeros.

La Asociación Nacional de Enfermeras Americanas (ANA) desarrolló un informe relacionado con los cuidados de enfermería para pacientes con alteraciones agudas, el cual identifica un grupo central de indicadores que incluye estructura, proceso y resultado; los indicadores de resultado abarcan la tasa de mortalidad, la duración de la estancia, los eventos adversos, las complicaciones y la satisfacción del paciente. ${ }^{2}$

Con base en lo anterior se concluye que uno de los aspectos relevantes como componente de la calidad del cuidado de enfermería es la satisfacción, cuya condición es imposible delimitar de manera precisa aun con una medida simple, ni es factible cuantificarla u observarla de manera directa; es necesario diseñar escalas de medición, las cuales para ser más objetivas requieren un proceso de validación, ${ }^{4}$ el cual constituye hoy una necesidad, con el fin de contar con herramientas más precisas para medir aspectos cualitativos.

En los proyectos de investigación cuando se requiere un instrumento para la recolección de información hay dos alternativas, construirlo o utilizar uno que 
haya sido aplicado en anteriores trabajos. Cuando se elige la segunda opción existe la posibilidad de contar con aquellos que han sido validados o no; aunque sí lo fue pero se realizó en un contexto o una población con características diferentes, es necesario realizar de nuevo el segundo paso de la construcción de un instrumento, el cual consiste en certificar que tiene ciertas características o atributos que hacen recomendable su utilización en el nuevo entorno.

Las características de las cuales depende la certificación de un instrumento son:

- Traducción y contratraducción.

- Prueba piloto de la versión adaptada.

- Validez de apariencia: parece medir lo que se intenta.

- Validez de constructo: no deja factores sin medir ni incluye dominios que no correspondan a la realidad.

- Validez de criterio: funciona de manera parecida a otros instrumentos utilizados para medir esa realidad que ya han sido certificados.

- Confiabilidad: su resultado se mantiene estable en un intervalo de tiempo corto (confiabilidad testretest) o sin importar si es aplicada por personas diferentes (confiabilidad interevaluador). Es decir, el resultado no varía dependiendo de las circunstancias externas.

- Sensibilidad al cambio: cuando la realidad que se está midiendo cambia, el instrumento puede detectar esa modificación.

- Utilidad: es práctico, fácil de aplicar y de procesar. $^{4,12,13}$

Con base en la complejidad del proceso descrito surge la inquietud ¿por qué validar un instrumento? La respuesta que reporta la literatura se sintetiza así: permite comparar la información ${ }^{14}$, puede resultar más económico y rápido que diseñar un nuevo instrumento y que al utilizar instrumentos mundialmente aceptados, pueden realizarse estudios entre diferentes países o culturas. ${ }^{4}$

Teniendo en cuenta estas consideraciones y la importancia que tiene la medición de la satisfacción para el sistema de seguridad social en Colombia, el grupo investigador realizó una búsqueda en diferentes bases de datos con el fin de detectar un instrumento que permitiera medirla en forma objetiva; se encontraron la escala CARE SAT de Patricia Larson cuyo propósito es permitir que los pacientes valoren el cuidado de enfermería ${ }^{15}$ y los catorce resultados del NOC. El grupo investigador revisó los aspectos de validez y especificidad de los instrumentos y tomó la decisión de realizar la validación de cuatro resultados del NOC, cuidados 3001, comunicación 3002, cumplimiento de las necesidades culturales 3004 y aspectos técnicos del cuidado 3013. Los resultados de la primera fase fueron publicados en Repertorio de Medicina y Cirugía, volumen $18, N^{\circ} 3$ de $2009^{1}$ y con esta base el paso a seguir era "la recolección de la información a la totalidad de la muestra", previa evaluación de los comités que rigen y evalúan el trabajo.

La investigación tiene por objeto validar los resultados del NOC cuya veracidad y confiabilidad ha sido aceptada en diferentes países del mundo, pero no en Colombia, aspecto determinante a la hora de hacer mediciones que permitan la toma de decisiones acertadas.

El NOC es el libro donde está consignada la clasificación de resultados de enfermería, la cual fue diseñada utilizando diversas fuentes enfermeras para identificar en forma inductiva los conceptos de resultados enfermeros, mediante un plan de obtención de datos que detalla los aspectos clínicos, ámbitos y grupo de edades de los pacientes. ${ }^{5}$ Por lo tanto, la clasificación NOC contiene resultados de los individuos, cuidadores, familia y sociedad que pueden utilizarse en todas las situaciones y especialidades clínicas, es resultado de la investigación dirigida por un equipo de estudiantes, facultativos y clínicos de diversas especialidades del Colegio de Enfermería de la Universidad de Iowa. Empezó en 1991 utilizando estrategias tanto cualitativas como cuantitativas. Los métodos consisten en análisis del contenido y del concepto, estudios de expertos, 
análisis de similitud, de agrupamiento jerárquico, organización multidimensional y del campo clínico, evaluando la exactitud entre puntuaciones, validez y utilidad de resultados en diez centros clínicos. La taxonomía tiene cinco niveles de resultados enfermeros acorde con el grado de abstracción: dominios (la mayoría abstractos), clases (nivel de abstracción medio alto), resultados (nivel de abstracción medio), indicadores (nivel de abstracción bajo) y nivel empírico (medida de las actividades de resultados). ${ }^{5}$ Los resultados dependen de las intervenciones enfermeras y describen el estado de los pacientes, la familia o la comunidad a un nivel conceptual.

Los resultados e indicadores son conceptos variables, permiten la determinación del resultado del paciente, la familia o la comunidad en cualquier momento de forma continua desde lo más negativo a lo más positivo en diferentes períodos de tiempo. El cambio en la puntuación puede explicarse como resultado de las intervenciones de enfermería y controlarse a lo largo del tiempo y en los centros de atención. ${ }^{2}$ La medición de los resultados valida si el paciente responde a las intervenciones de enfermería proporcionadas y facilita los datos necesarios para determinar la efectividad de estas, el momento adecuado para medir los resultados variará porque algunas intervenciones generan respuestas muy rápido, mientras en otras se espera la respuesta del paciente en un largo período. Con 330 resultados es difícil seleccionar los específicos para un tipo de práctica determinada, una forma de hacerlo es que las enfermeras puedan identificar una lista de resultados que utilizan a diario con sus pacientes; una segunda es revisar la lista de resultados utilizados por organizaciones especializadas. ${ }^{16}$

La clasificación actual está constituida por siete dominios, 29 clases, 330 resultados con sus respectivos indicadores y dado que los resultados son conceptos variables que representan estados, es esencial un método para medir los conceptos, por lo que se utiliza una escala tipo likert de cinco puntos. En esta edición se cuenta con once escalas identificadas por letras del alfabeto dentro de las que se encuentra la escala s, que es nueva y se utiliza para puntuar la satisfacción del paciente en varios conceptos. Las unidades de medida van desde no del todo satisfecho hasta completamente satisfecho. ${ }^{16}$ Los dominios descritos por el NOC son:

\section{Salud funcional.}

2. Salud fisiológica.

3. Salud psicosocial.

4. Conocimiento y conducta de salud.

5. Salud percibida que incluye la clase de satisfacción con los cuidados, conformada por 14 resultados.

6. Salud familiar.

7. Salud comunitaria. ${ }^{1,6}$

Uno de los aspectos relevantes en la medición de la calidad es la satisfacción del usuario, perspectiva muy importante teniendo en cuenta que en salud no solo se deben ofrecer condiciones básicas de capacidad tecnológica y científica, de suficiencia patrimonial y financiera, y de capacidad técnico-administrativa (categorías evaluadoras de calidad); este atributo en la rama de los servicios también está determinado por la relación del equipo de salud que en el presente estudio corresponde a la establecida entre el personal de enfermería con el paciente/usuario, a quien en adelante llamaremos paciente. Este vínculo genera calidad y cantidad de atención, de lo cual depende en gran medida el grado de satisfacción con el servicio ofrecido.

El NOC define satisfacción con los cuidados como resultados que describen percepciones de un individuo de la calidad y la adecuación de su asistencia sanitaria $^{6}$, y paciente como el destinatario de los cuidados aunque reconoce que puede denominarse cliente o usuario en algunos contextos. Para el objetivo de esta investigación, el grupo adoptó esta definición de satisfacción ubicado en el contexto (para pacientes hospitalizados en UCI), como el constructo a ser evaluado. ${ }^{1}$

La publicación oficial de la Asociación Médica Americana expresa: "Las unidades de cuidados intensivos (UCI) son departamentos de los hospitales con equipamiento especializado y personal altamente cualificado 
para tratar a pacientes con una enfermedad o traumatismo grave. Los pacientes pueden ser ingresados en la UCI desde el servicio de urgencias u otro departamento del hospital, después de una intervención quirúrgica o ser trasladados allí desde otro centro sanitario...". ${ }^{17}$

El concepto de enfermería ha evolucionado a partir del tiempo, la primera en expresarlo fue Florence Nightingale en su libro Notas de enfermería que señala "enfermería es cuidar y ayudar al paciente que sufre de una enfermedad a vivir; lo mismo que la enfermería de la salud es mantener o lograr que la salud de cualquier niño o persona sana se mantenga y no sea susceptible a la enfermedad". Agrega Nightingale que lo que la enfermera debe lograr es llevar al paciente a tal estado que la naturaleza, de manera simple pueda actuar en él. De otros enunciados que se toman como referencia para el presente trabajo está el de Meleis (1985), quien afirma "la enfermera interactúa en la situación del cuidado de la salud con el ser humano, siendo parte integral de su contexto sociocultural y que está en una forma de transición o anticipación; las interacciones del paciente-enfermera están organizadas alrededor de un propósito y esta última utiliza acciones deliberadas para reforzar, causar o facilitar la situación de salud". En 1998 Watson la define como "es el conocimiento, valores, filosofía, dedicación y acciones relacionadas con las transacciones y subjetividad del cuidado humano. Como tal, el cuidado es el ideal moral de enfermería y consiste en el intento transpersonal de proteger, reforzar y preservar la dignidad de la persona, ayudándola a encontrar sentido a la existencia, aun en el sufrimiento y aumentar su autoconocimiento, control y bienestar con sentido de armonía interna, a pesar de las circunstancias externas". ${ }^{18}$

Por otro lado el cuidado se ha definido como la razón de ser de la enfermería y su objeto central de estudio. En Colombia la Ley 911 de 2004 define el acto de cuidar como el ser y esencia del ejercicio de la profesión. Se fundamenta en sus propias teorías y tecnologías, y en conocimientos actualizados de las ciencias biológicas, sociales y humanísticas. Se da a partir de la comunicación y relación interpersonal humanizada entre el profesional de enfermería y el ser humano, sujeto de cuidado, la familia o grupo social, en las distintas etapas de la vida, situación de salud y del entorno. Implica un juicio de valor y un proceso dinámico y participativo para identificar y dar prioridad a las necesidades y decidir el plan de cuidado de enfermería, con el propósito de promover la vida, prevenir la enfermedad, intervenir en el tratamiento, en la rehabilitación y dar cuidado paliativo con el fin de desarrollar, en lo posible, las potencialidades individuales y colectivas. ${ }^{19}$

Como lo plantea Liliana Villarraga, cuidar implica "ofrecer a la persona y su familia el cuidado comprometido, interesado, silencioso, directo y cálido, basado en el conocimiento y la seguridad que requiere cada persona en su experiencia en un momento dado, de acuerdo con su propia historia, su familia, su contexto psicosocial y cultural en los momentos críticos de la vida, pérdida, separación y muerte". ${ }^{20}$

Enmarcados en los conceptos anteriores, un grupo investigador realizó en el año 2007 la validez de apariencia de los catorce resultados del NOC que evalúan la satisfacción del paciente usuario, conformado por expertos como fueron un médico candidato a magíster en Epidemiología, una enfermera magíster en Administración en salud y tres enfermeras con más de dos años de experiencia de los cuales mínimo uno debía ser en UCI. Teniendo en cuenta la pertinencia, la utilidad y la claridad de los ítems, seleccionaron cuatro resultados: cuidados (3001), comunicación (3002), cumplimiento de las necesidades culturales (3004) y aspectos técnicos del cuidado (3013). Con base en esta selección aplicaron una prueba piloto en el año 2008 con el fin de validar un instrumento para medir la satisfacción ${ }^{1}$ con el objetivo de determinar la adaptación transcultural y validez de apariencia. Durante su análisis se consideró que era necesario además del análisis de expertos, realizar una concordancia de la validez de apariencia mediante la presentación de los catorce resultados acompañados de un instrumento autodirigido, en el cual los participantes señalaban cuál consideraban pertinente y útil para medir la satisfacción del paciente con el cuidado de enfermería en UCI, haciendo claridad que el resultado no se podía disgregar. El proceso se realizó con un grupo de personas que tuviera una de las siguientes condiciones: 
ser familiar de paciente que hubiese estado en la UCI por un período mayor de 48 horas, haber estado hospitalizado en la UCI por más de 48 horas, ser médico(a) o enfermero(a) con especialización en atención a pacientes críticos con experiencia en UCI.

\section{Métodos}

Para verificar la concordancia de validez de apariencia se incluyeron siete personas, dos médicos intensivistas, dos enfermeras especialistas en cuidado crítico con experiencia en UCI, dos pacientes y un familiar de paciente de la UCI. La selección de estas personas fue aleatoria entre una lista de candidatos que aceptaron responder los datos requeridos.

Teniendo en cuenta las características disímiles de los integrantes y la dificultad para conciliar una reunión por las diversas actividades de los mismos que hacían incompatibles los horarios, los investigadores decidieron diseñar un instrumento autodiligenciado con su respectivo instructivo, el cual se aplicó durante febrero de 2009, asumiendo el rol que desempeñan o desempeñaron; como precaución se aceptó que no hubieran tenido contacto con el anterior estudio realizado por Aponte y Carrillo. El objetivo de esta fase del trabajo consistía en identificar cuáles de los 14 resultados del NOC se consideran pertinentes para determinar la satisfacción del usuario con el cuidado de enfermería en la UCI. Con base en los criterios establecidos por Moriyama los miembros del grupo debían tener en cuenta si cada resultado era:

- Razonable y comprensible: cuando las preguntas de cada ítem resultan entendibles para un sujeto promedio y con facultad mental adecuada.

- Sensible a variaciones en el fenómeno que se mide: si las preguntas de cada ítem y el resultado en su conjunto podrían separar sujetos con diferentes niveles de satisfacción.

- Con suposiciones básicas justificables o por intuición razonables, es decir, si existe justificación para la inclusión de cada resultado.
- Con componentes definidos, se refiere a la claridad de los términos o ítems de cada resultado.

- Derivables de datos factibles de obtener, es decir, la posibilidad de obtener información sobre satisfacción a partir de las respuestas del paciente.,21

El grupo conformado por dos médicos, dos enfermeras, dos pacientes y un familiar de paciente que estuvo hospitalizado en UCI recibieron un instrumento que contenía los catorce resultados para que, previa explicación de la manera de diligenciarlo por parte de los investigadores, fueran seleccionados aquellos que cumplían con las características solicitadas de satisfacción y devueltos para realizar su respectiva tabulación y análisis (Anexo 1).

La Tabla 1 muestra los resultados de las respuestas de cada uno de los participantes en el proceso de validez de apariencia para evaluar la aplicabilidad de resultados del NOC en la clase de satisfacción, en lo relativo al cuidado de enfermería del paciente hospitalizado en UCI.

\section{Resultados}

La validez de apariencia del instrumento a utilizar para evaluar la satisfacción había sido sometido por Aponte y Carrillo a un consenso de expertos (comité del grupo investigador: cinco personas entre los cuales hay especialistas en enfermería de cuidado intensivo, magíster en administración en salud y un candidato a magíster en Epidemiología clínica), que concluyó que la satisfacción del paciente hospitalizado en UCI podía ser medida de manera adecuada a través de cuatro resultados de los catorce propuestos por la NOC, los cuales según los autores pueden utilizarse en forma independiente: ${ }^{2}$ cuidados (3001), comunicación (3002), cumplimiento de las necesidades culturales (3004) y aspectos técnicos del cuidado (3013). Los resultados de la prueba piloto condujeron a realizar un sondeo entre personas de la UCI, con el fin de dar mayor validez de apariencia.

En el actual proceso para validez de apariencia el instrumento autoadministrado fue respondido en forma individual. Teniendo en cuenta las diversas caracterís- 
ticas de las personas incluidas en el proceso, el grupo investigador determinó que se consideraría válido el resultado seleccionado por cinco de siete personas, por encima del punto medio, con el fin de estar lo más cerca posible al consenso. El análisis y tabulación de los datos con el criterio descrito seleccionó tres de los catorce resultados (Tabla 1), dos de los cuales habían sido seleccionados por el grupo de expertos anterior, cuidados (3001) y comunicación (3002). El tercero correspondiente a seguridad fue escogido por los siete participantes, excluyendo por lo tanto dos de los cuatro del estudio anterior e incluyendo uno nuevo. Es por esta causa que la concordancia entre los datos arrojados es importante para dar mayor validez de apariencia a los resultados generados por el trabajo de investigación.

Respecto al familiar y los pacientes que tuvieron experiencia en UCI, en el momento de la aplicación del instrumento fueron muy receptivos con el proceso "ya que estar o tener a alguien en la UCI representa una situación difícil" y por tal motivo querían contribuir a que el cuidado en esta situación se mejorara y otras personas no pasaran por situaciones difíciles evitables; llama la atención un paciente con un nivel de escolaridad bajo a quien se le dificultó el proceso pero demostró compromiso para realizarlo, el grupo investigador piensa que es una razón más por la cual es conveniente un instrumento con el menor número posible de preguntas. En este caso el paciente demostró cansancio, tuvo que desarrollarla en varios momentos y manifestó en forma verbal que el resultado que más le gustó fue el de seguridad.

\section{Discusión}

En la atención en salud y especial en la calidad del cuidado de enfermería, la satisfacción del paciente es relevante por ser un concepto subjetivo y es necesario contar con instrumentos validados que permitan acercarse a la realidad. La validez de apariencia de los catorce resultados del NOC permitió identificar que los tres que tienen mayor aplicabilidad para medir la satisfacción del paciente hospitalizado en UCI con la atención en enfermería son: cuidados (3001) y comu- nicación (3002), lo que ratifica la selección hecha por los expertos descrita por Aponte y Carrillo' además de seguridad (3010) que no había sido incluida pero en el presente estudio lo fue por la totalidad de los integrantes del grupo.

El resultado denominado comunicación (3002) mide uno de los conceptos pertenecientes a la definición de enfermería hecha por Meleis cuando habla de la interacción paciente-enfermera y por Watson al hacer énfasis en las acciones relacionadas con las transacciones y subjetividad del cuidado humano. Ratifica lo encontrado por Pérez y Taboada en el trabajo sobre Necesidades de la familia frente a la hospitalización del paciente en UCI, Hospital de San José, que describe las situaciones que generan mayor angustia en la familia y las necesidades de esta frente a la hospitalización del paciente en UCI, como información, apoyo y comodidad, dan lugar a estrategias que permiten lograr satisfacción con la atención brindada. ${ }^{22}$

El análisis del resultado cuidados (3001) es coherente con lo encontrado por Rodríguez y Romero en el trabajo denominado El cuidado que brinda la enfermera jefe al paciente en la UCI de la Clínica San Pedro Claver, donde concluye que es dirigido en forma responsable, ordenada, rápida, oportuna, con calidad humana, integrando acciones tanto para el paciente como para la familia y se desarrolla teniendo en cuenta aspectos observados por la familia como prioritarios. ${ }^{23}$

El resultado concerniente a seguridad permite medir el concepto de proteger expresado por Watson en su definición de enfermería. A su vez los ítems del resultado cuidados, valoran los aspectos relacionados con la definición de enfermería de Meleis y Watson y del acto de cuidado de enfermería objeto de la Ley 911 de 2004 de la República de Colombia.

Los tres resultados seleccionados son coherentes con lo referenciado por Ariza en su artículo “¿Es posible humanizar la atención de enfermería en el paciente en estado crítico?". Allí consigna los hallazgos de los doctores Lázaro Silva Ramos y Nelson Martínez, cuando indagaron sobre la satisfacción de la familia con el cuidado y la atención que se brinda en el área 
intensiva municipal, concluyendo que cuando el equipo de salud de una UCI brinda seguridad, confianza, ánimo y apoyo emocional unido a explicaciones claras pero sencillas de la situación y evolución del enfermo, resulta muy importante, reduciendo la ansiedad y favoreciendo el grado de satisfacción tanto de la familia como del paciente. ${ }^{24}$

Teniendo en cuenta los resultados del proceso de validación realizado por Aponte y Carrillo que incluía los cuatro resultados obtenidos en el consenso de expertos el número de ítems fue considerado como un elemento importante desde el punto de vista de la utilidad del instrumento. Una de las observaciones de un encuesta- dor y la coordinadora del mencionado estudio, fue que por lo extenso había sido de difícil aplicación en un paciente y otro decidió no continuar, con lo cual el instrumento actual disminuye en un $25 \%$ dicho número.

Las enfermeras consideraron que uno de los ítems que conforman cada uno de los resultados no era del todo bien entendido o útil, pero realizaron la elección teniendo en cuenta las condiciones de la encuesta la cual aclara que se debe seleccionar todo el resultado. Las enfermeras estuvieron de acuerdo en que uno de los resultados más importantes es el de seguridad y lo consideran el más aplicable en el proceso de medir la satisfacción.

Tabla 1: Evaluación de validez de apariencia

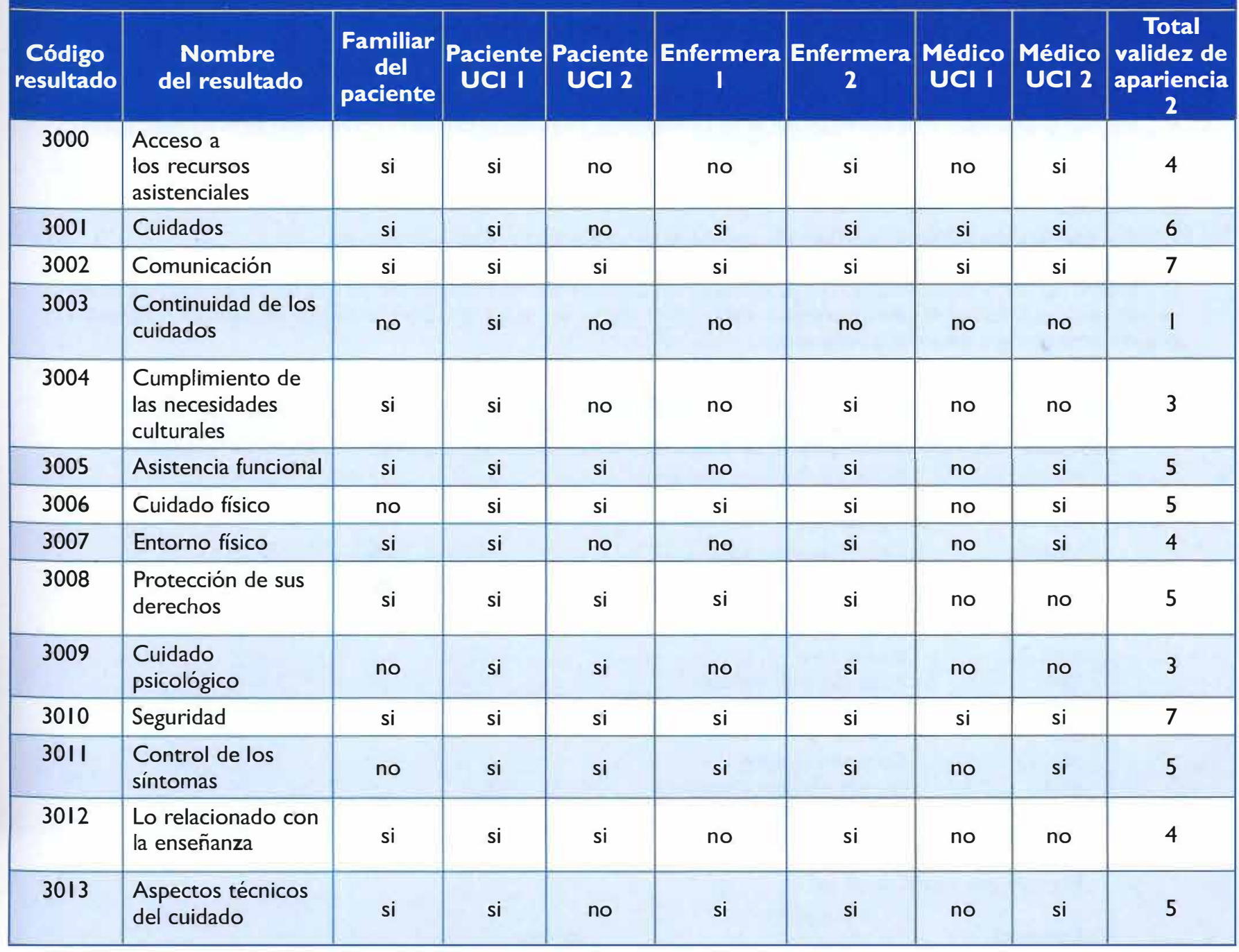




\section{Anexo 1 \\ INSTRUMENTO PARA LA EVALUACIÓN DE LA VALIDEZ DE APARIENCIA DE LA SATISFACCIÓN DEL PACIENTE/USUARIO CON EL CUIDADO DE ENFERMERÍA EN LA UNIDAD DE CUIDADOS INTENSIVOS}

NOMBRE: PROFESIÓN:

\section{FECHA:}

OBJETIVO: identificar cuáles de los 14 resultados del NOC (clasificación de resultados de enfermería) que miden satisfacción, son pertinentes para determinar la satisfacción del usuario con el cuidado de enfermería en la unidad de cuidado intensivo.

La clasificación de resultados de enfermería (NOC, por sus siglas en inglés) evalúa la satisfacción con 14 resultados, compuestos por diferentes ítems de los cuales el grupo investigador se propone validar lo correspondiente a satisfacción del paciente/usuario con el cuidado de enfermería en la Unidad de Cuidados Intensivos.

Los resultados descritos son: acceso a los recursos asistenciales (3000), cuidados (3001), comunicación (3002), continuidad de los cuidados (3003), cumplimiento de las necesidades culturales (3004), asistencia funcional (3005), cuidado físico (3006), entorno físico (3007), protección de sus derechos (3008), cuidado psicológico (3009), seguridad (3010), control de los síntomas (3011), lo relacionado con la enseñanza (3012) y aspectos técnicos del cuidado (3013). Es de tener en cuenta que por aspectos metodológicos un resultado no puede ser fraccionado, debe ser aplicado en su totalidad.

A continuación usted encuentra los catorce resultados, léalos cuidadosamente teniendo en cuenta si es:

- Razonable y comprensible: si las preguntas de cada ítem resultan entendibles para un sujeto promedio y con facultad mental adecuada.

- Sensible a variaciones en el fenómeno que se mide: cuando las preguntas de cada ítem y el resultado en su conjunto podrían separar sujetos con diferentes niveles de satisfacción.

- Con suposiciones básicas justificables o por intuición razonables, es decir, si existe justificación para la inclusión de cada resultado.

- Con componentes definidos, se refiere a la claridad de los términos o ítems de cada resultado.

Con base en su juicio y experiencia seleccione los que en su conjunto permitan evaluar la satisfacción del paciente con el cuidado de enfermería en la unidad de cuidado intensivo. Para hacerlo marque con una X el Sí frente al nombre del resultado o resultados elegidos en la lista que encuentra a continuación.

\begin{tabular}{|l|l|l|}
\hline Código & \multicolumn{1}{|c|}{ Resultado } & Sí \\
\hline 3000 & Acceso a los recursos asistenciales & \\
3001 & Cuidados & \\
3002 & Comunicación & \\
3003 & Continuidad de los cuidados & \\
3004 & Cumplimiento de las necesidades culturales & \\
3005 & Asistencia funcional & \\
3006 & Cuidado físico & \\
3007 & Entorno físico & \\
3008 & Protección de sus derechos & \\
3009 & Cuidado psicológico & \\
3010 & Seguridad & \\
3011 & Control de los sintomas & \\
3012 & Lo relacionado con la enseñanza & \\
3013 & Aspectos técnicos del cuidado & \\
\hline
\end{tabular}

Marque con una $X$ si ud. es:

Paciente: Médico:

Familiar de paciente: Enfermera: 


\section{Referencias}

1. Aponte J, Carrillo A, Lizarazo C, Silva O. Satisfacción del cuidado de enfermería en UCI. Repertorio de Medicina y Cirugía. 2009;18(3):152-60.

2. Moorhead S, Johnson M, Maas M. Clasificación de resultados de enfermería (NOC). Tercera ed. Madrid: Elsevier - Mosby. 2005.

3. Moriyama I. Indicators of social change. In Bernert Sheldon E, (ed.). Problems in the measurements of health status. New York: Russell Sage Foundation. $1968 ; 573-600$

4. Sánchez R, Echeverry J. Validación de escalas de medición en salud. Revista de Salud Pública. 2004 Nov;6(3).

5. Johnson M, Maas M, Moorhead S. Investigación de los resultados de los pacientes dependientes de enfermería: Fases I y II. En Johnson M, Maas M, Moorhead S, (eds.). Clasificación de resultados de enfermería (CRE). Madrid, España: Mosby - Elsevier. 2003;22-43.

6. Moorhead S, Johnson M, Maas M. Introducción a la taxonomía de la NOC. En Moorhead S, Johnson M, Maas M, (eds.). Clasificación de resultados de enfer mería (NOC). Tercera ed. Madrid: Elsevier - Mosby. 2005;104-26.

7. Cabrales R. Validez de instrumentos de medición sobre acoso laboral en médicos. Revista Médica de Risaralda. 2008;14(1):36-45. Ref Type: Joumal (Full).

8. Lang N, Marek K. Clasification of Patient Outcomes. Joumal of Professional Nursing. 1990;6,153-163. Ref Type: Joumal (Full).

9. Salive M, Mayfield J, Weissman W. Patient Outcomes Research Teams and the Agency for Health Care Policy and Research. Health Services Research. 1990;25(5),697-708. Ref Type: Joumal (Full).

10. Aydelotte, M. The Use of patient Welfare as a criterion measure. Nursing Research. 2011;11:10-4.

11. Bader K. Patient Care Report Cards: An Analysis. Outcomes. Management for Nursing Practice. 1998;2(1),29-36. Ref Type: Journal (Full).
12. Arribas A. Adaptación transcultural de instrumentos, guía para el proceso de validación de instrumentos tipo encuestas. Revista Científica de la AMBB. 2006;16(3):74-82

13. Pinto A. Indicadores de cuidado. Actualizaciones en enfermería. 2001 Mar;4(1):13-8.

14. Arribas M. Diseño y validación de cuestionarios. Matrona profesión. 2004;5(17).

15. Ariza O. Satisfacción del cuidado de enfermería en el paciente cardiaco. MEDWAVE. 2004 Apr.

16. Moorhead S, Johnson M, Maas M. Clasificación Actual. In Moorhead S, Johnson M, Maas M, (eds.). Clasificación de resultados de enfermería (NOC). Tercera ed. Madrid: Elsevier - Mosby. 2005;20-50.

17. Institutos Nacionales de Salud. Terapia intensiva. http://www nlm nih gov/medlineplus/spanish/criticalcare html 2011.

18. Duran de Villalobos M. Enfermería desarrollo teórico e investigativo. Universidad Nacional de Colombia. 1998.

19. Responsabilidad deontológica para el ejercicio de la profesión de enfermería en Colombia, Ley 911, Congreso de Colombia. 2004.

20. Villarraga L. Dimensiones del cuidado. El poder del cuidado generador del conocimiento en enfermería. Bogotá: Universidad Nacional de Colombia. 1998;109.

21. López Pumar G, Del Castillo N, Oramas A. Validez y confiabilidad del cuestion ario. Índice de Capacidad de Trabajo (ICT) en su versión cubana. Revista Cubana de Salud y Trabajo, 2011;12(2),29-34. Ref Type: Journal (Full)

22. Pérez MM, Taboada H. Necesidades de la familia frente a la hospitalización del paciente en UCI Hospital de San José. Bogotá DC: Fundación Universitaria de Ciencias de la Salud. 1999.

23. Rodríguez $\mathrm{D}$, Romero $\mathrm{M}$. Cuidado que brinda la enfermera al paciente de la UCI desde la mirada de la familia. Bogotá DC: Fundación Universitaria de Ciencias de la Salud. 2003.

24. Ariza C. ¿Es posible humanizar la atención de enfermería al paciente en estado crítico? Actualizaciones de enfermería. 1998;1:18-20. 